\title{
Primary Cerebellopontine Angle Melanocytoma: Review
}

\author{
Isaac Phang, M.B.B.S., M.R.C.S. ${ }^{1}$ Rahim Elashaal, M.B.B.S., M.R.C.S. ${ }^{1}$ \\ James Ironside, M.B.B.S., F.R.C.Path. ${ }^{2}$ Sam Eljamel, M.D., F.R.C. S. (Ed,Ir,SN). ${ }^{1}$ \\ ${ }^{1}$ Department of Neurosurgery, Ninewells Hospital and Medical School, \\ Dundee, Scotland, United Kingdom \\ 2 Department of Neuropathology, Western General Hospital, \\ Edinburgh, Scotland, United Kingdom \\ Address for correspondence and reprint requests Sam Eljamel, M.D., \\ F.R.C.S. (Ed,Ir,SN), Professor, Consultant Neurosurgeon, Ninewells \\ Hospital and Medical School, Dundee DD1 9SY, Scotland, UK \\ (e-mail: m.s.eljamel@dundee.ac.uk).
}

J Neurol Surg Rep 2012;73:25-31.

\begin{abstract}
Introduction Primary cerebellopontine angle melanocytomas (PCPAMs) are very rare. Their natural history and prognosis are not fully understood. We reviewed the literature and add a new case to analyze PCPAM's presentation, radiological features, and outcome of treatment.

Methods We performed a literature review using Medline, Embase, PubMed, and Cochrane databases. We searched for melanocytoma, melanoma, and pigmented tumors in the posterior cranial fossa and CPA to identify PCPAM. We have also searched our institution's neuro-oncology database.

Results We identified 23 PCPAM from the literature and one case of our own. The mean age at presentation was 44.4 years with slight male preponderance. PCPAM presented with cerebellopontine angle (CPA) syndrome with or without hydrocephalus. Preoperative diagnosis was difficult; they appeared hyperintense on $\mathrm{T} 1$ and isointense on T2 magnetic resonance imaging (MRI) and enhanced with gadolinium. However, the final diagnosis was only made by immunohistochemical examination. Total surgical resection of PCPAM was associated with prolonged survival while subtotal excision was

Keywords

- CPA

- melanocytoma

- pigmented tumors associated with frequent recurrence.

Conclusion PCPAM are very rare and should be considered in the differential diagnosis of all CPA lesions that appear hyperintense on T1 and isointense on T2 MRI images. Patients with PCPAM should undergo total surgical resection to avoid fatal recurrences.
\end{abstract}

Primary pigmented tumors in the cerebellopontine angle (CPA) are uncommon and include pigmented meningiomas, melanocytic colonization of meningothelial meningioma, malignant melanomas, meningeal melanocytomas, melanotic schwannomas, and melanoblastosis. The differential diagnosis is often confusing owing to their similar appearance on preoperative investigations and similar histological features. Hence, confirmation by electron microscopy (EM) and immunohistochemical tests are required. As the biological behavior, treatment, and prognosis of these lesions vary markedly, it is important to make the correct pathologic diagnosis.
Melanocytomas are slow-growing tumors of melanocytes. They compress rather than infiltrate adjacent tissues. Melanocytes are normally found in human leptomeninges, ${ }^{1}$ and are thought to originate from the neural crest found within the basal layer of the epidermis and the leptomeninges covering the base of the brain and the brainstem. ${ }^{2-4}$ Consequently, pigmented intracranial tumors most commonly involve pons, cerebellum, cerebral peduncles, medulla, interpeduncular fossa, and inferior surfaces of the cerebrum. ${ }^{3-5}$ These neoplasms are generally divided into diffuse melanosis, meningeal melanocytomas, and primary malignant received

June 30, 2011

accepted after revision

December 5, 2011

published online

April 20, 2012
Copyright (c) 2012 by Thieme Medical Publishers, Inc., 333 Seventh Avenue, New York, NY 10001, USA. Tel: +1(212) 584-4662.
DOI http://dx.doi.org/ 10.1055/s-0032-1311756. ISSN 2193-6358. 
melanomas. Limas and $\mathrm{Tio}^{2}$ coined the term meningeal melanocytoma after observing melanosomes and premelanosomes in tumor cells of a heavily pigmented foramen magnum tumor. Primary melanocytomas have been reported to be more common in men. We add another case and review the literature to establish clinical, radiological and histopathological features, treatment, and prognosis of these rare tumors.

\section{Methods}

We reviewed Medline, Embase, PubMed, and the Cochrane databases to identify all patients who had rimary cerebellopontine angle melanocytoma (PCPAM). We searched the following terms: melanocytoma, pigmented tumors, and melanoma combined with posterior cranial fossa or CPA. We included only those tumors that fulfilled the diagnostic criteria for primary melanocytoma and those located in the CPA. The clinical presentation, radiological features, histological findings, treatment, and the outcome of the treatment of these patients were collated and analyzed to document PCPAM's behavior and outcome.

We also reviewed our neuro-oncology and neuropathology databases to look for any PCPAM in our institution.

The collated data were analyzed using simple statistics to determine frequencies, means, medians, and rates.

\section{Case Report}

A 40-year-old man presented with occipital headaches associated with nausea. On examination he displayed truncal ataxia, The new clinical signs were sensory neural deafness on the right and ataxia. His magnetic resonance imaging (MRI) brain scan demonstrated a solitary lesion arising in the right para-pontine space and CPA. It appeared to be extraaxial with mass effect and normal internal auditory meatus appearance. On $\mathrm{T} 2 \mathrm{~W}$ the lesion was mixed appearance: isointense, hyperintense, and areas of hypointensity ( -Fig. 1A). On T1W the lesion appeared hyperintense with areas of isointensity (-Fig. 1B). The lesion enhanced after gadolinium administration (-Fig. 1C).

\section{Operation}

The patient underwent a posterior fossa craniotomy. The craniotomy was performed via retro-sigmoid approach with the intent of total removal. The seventh cranial nerve was monitored during surgery but the eighth was not monitored because he was profoundly deaf on the right. At operation a purplish-pink friable tumor involving the right CPA, extending down to the foramen magnum inferiorly was found. This was dissected gradually and removed using an ultrasonic dissector and microsurgery. However, the tumor was found to be invading the brainstem and the surrounding structures, encasing the right vertebral artery. Hence only subtotal excision was possible. Postoperatively, the patient had excellent postoperative recovery with Karnofsky performance score of 80 . He proceeded to 4 -week course of radiotherapy and was seen in the clinic with satisfactory
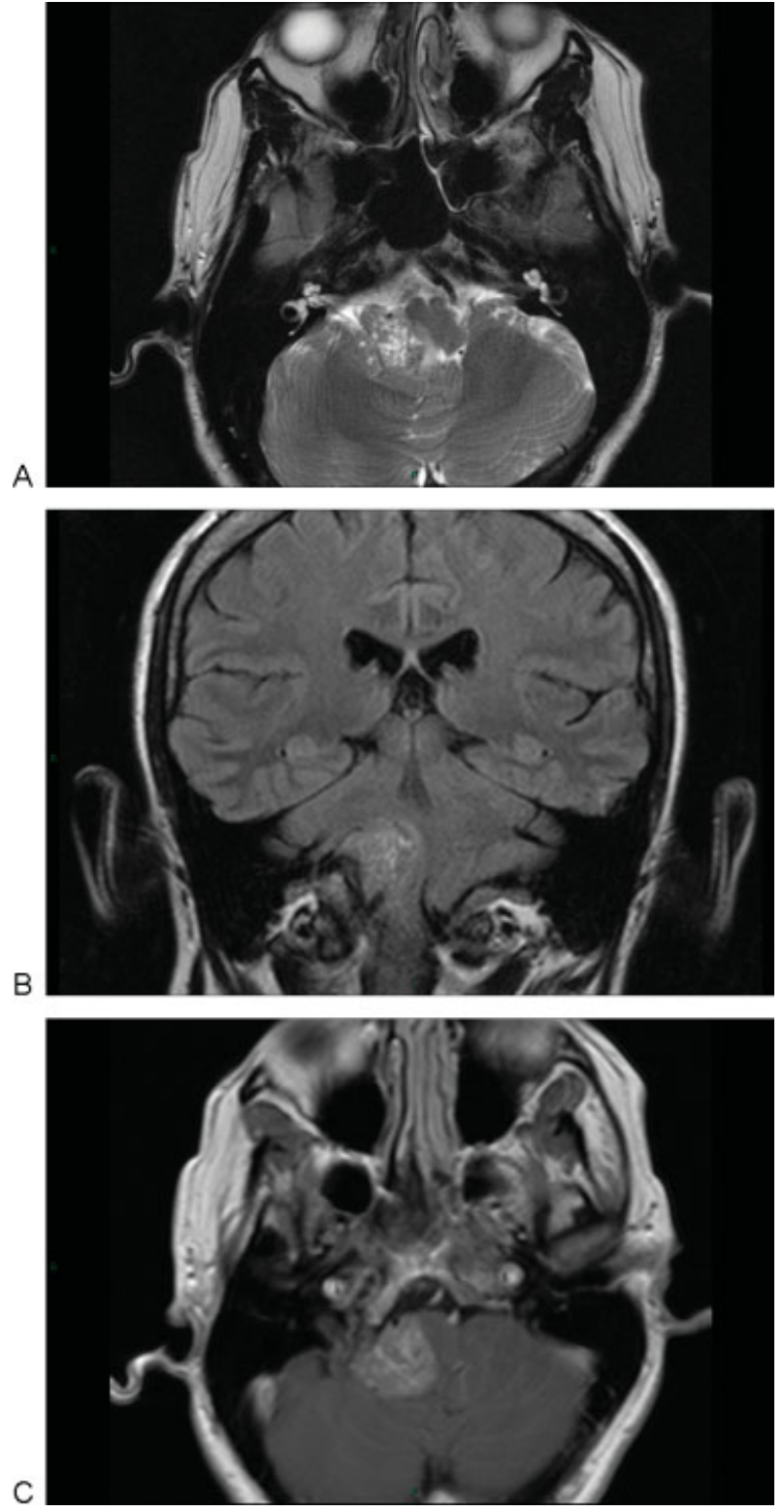

Figure 1 Magnetic resonance imaging scan of primary cerebellopontine angle melanocytoma. (A) Axial T2 image showing a right cerebellopontine angle (CPA) isointense mass and areas of hypo and hyper intensity. (B) Coronal T1 image showing a right CPA mass displacing the brainstem. The lesion was slightly hyperintense with areas of isointensity. (C) Axial T1 with contrast demonstrating mild homogeneous enhancement.

postoperative progress. Histologically tumor cells appeared relatively uniform, and were arranged in solid lobules with no evidence of necrosis. The nuclei were rounded and exhibited a mild degree of pleomorphism, with rounded nucleoli (-Fig. 2A). Granular brown pigment was present in occasional tumor cells and in macrophages around the tumor lobules that gave a positive reaction for melanin on a Singh stain. Immunohistochemistry for Melan-A (-Fig. 2B), HMB45, and neurone-specific enolase was strongly positive. A stain for S-100 protein was weakly positive. These findings indicated that this was a melanocytic lesion, rather than a melanotic 
A

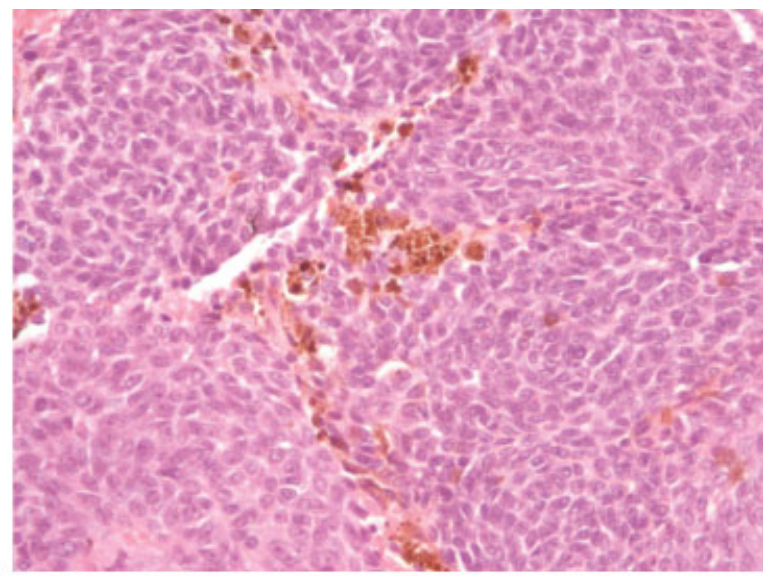

B
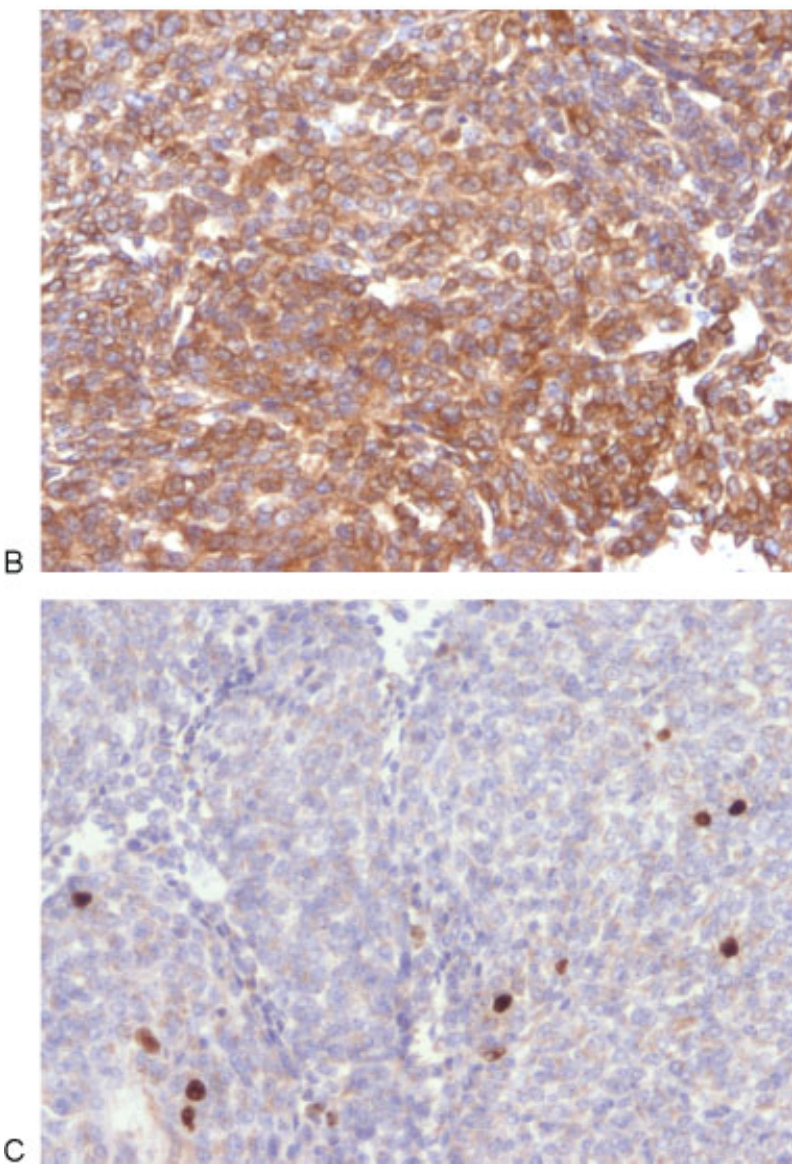

Figure 2 Histological features of melanocytoma. All original magnifications are $x 200$. (A) Hematoxylin and eosin stain demonstrating solid lobules of uniform cells with scanty pigment (mostly in macrophages) and rounded nuclei. (B) Immunocytochemistry for Melan-A showing strong cytoplasmic positivity, confirming the melanocytic nature and helping to exclude meningioma and schwannoma.

(C) Immunocytochemistry for $\mathrm{K}_{\mathrm{i}}-67$ revealing a low proliferation index $(\sim 5 \%)$ which would be unusual in a primary or metastatic malignant melanoma.

Schwannoma. Mitotic activity was not a prominent feature $(<1 \%)$ and the cell proliferation index on a $\mathrm{K}_{\mathrm{i}}-67$ immunostain was low $(<5 \%)$ ( $>$ Fig. 2C). The relative lack of mitotic activity, necrosis, and pleomorphism, along with a low cellular proliferation index, favored a diagnosis of melanocytoma rather than malignant melanoma. After 3.5 years of initial presentation, tumor progression was detected on MRI surveillance and further surgery was undertaken. Histological analysis of the second specimen demonstrated similar appearances to the initial specimen (few mitotic figures $(<1 \%)$ and a $\mathrm{K}_{\mathrm{i}}-67$ proliferation index of $<5 \%$ ), with no evidence of malignant transformation. However, the patient succumbed to unrelated causes 6 weeks later.

\section{Results}

We collected 23 PCPAM from the literature and one PCPAM from our own database. There were enough data on each case to include in our analysis (-Table $\mathbf{1}$ ). The mean age at presentation was 44.4 years (range: 9 to 71 years), men were affected more commonly than women 7:5. Between the ages of 30 to 60 years men were affected twice as women.

The most common presenting symptom was headache (58\%), dysphagia, ataxia, and vomiting was noted in $29 \%$, diplopia and sensory deafness in $25 \%$, facial numbness, papilledema and leg weakness in $21 \%$, neck pain in $8 \%$, and syncope in $4 \%$. The median duration of symptoms was 5 months (range: few weeks to 14 years).

MRI characteristically demonstrated hyperintensity on T1-weighted images and hypo- or isointensity on T2-weighted images. These tumors usually enhanced homogenously with gadolinium. Histologically, PCPAM appeared as shown in - Fig. 2.

Twelve patients had total surgical resection (11 in one stage and 1 in two stages) with median follow-up of 4 years with no deaths. Early recurrence was reported at 8 months in one case ( $8 \%)$. Late recurrence was reported in two cases: one after 8 years and the other after 10.8 years. All three recurrences died within 12 months from recurrence irrespective of treatment. Only 1 of 12 totally resected PCPAM had adjuvant radiotherapy. Nine patients had subtotal removal. Five patients had subtotal removal with 1 month to 3.5 years followup with 1 early death (20\%), two progressed within months (40\%),and one died within a year (20\%). A total of $80 \%$ of subtotal resections either progressed or died within 12 months of diagnosis. One subtotal resection had no follow-up information. Three had subtotal resection followed by radiotherapy; one died after 6 months due to hemorrhage, one had no recurrence at 2 years and the third recurred at 3 years and died at 3.5 years. One patient only had biopsy and died within 3 days of surgery, and one patient had diagnosis made at autopsy.

\section{Discussion}

Primary CPA melanocytomas are very rare tumors and it is unlikely one center or one team will have sufficient numbers to establish their behavior, prognosis, or best treatment. Our review revealed peak incidence between 30 and 60 years of age and preponderance in males, but they can present at any age. The duration of symptoms varied widely, from 3 months to 14 years. PCPAM presented with symptoms and signs of an expanding mass in the posterior cranial fossa, and may lead to 


\begin{tabular}{|c|c|c|c|c|c|c|c|c|c|c|c|c|}
\hline 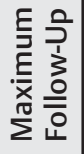 & $\stackrel{\vec{n}}{\stackrel{n}{m}}$ & $\begin{array}{l}E \\
m\end{array}$ & $\mid$\begin{tabular}{l}
$\overrightarrow{1}$ \\
$\infty$ \\
0 \\
\hdashline
\end{tabular} & 1 & $\begin{array}{l}E \\
\infty \\
\infty\end{array}$ & 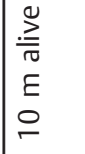 & 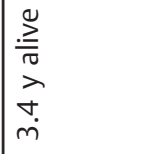 & 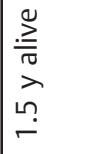 & 岕 & $\begin{array}{l}3 \\
m\end{array}$ & $\vec{a}$ & 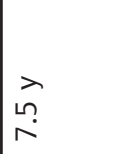 \\
\hline 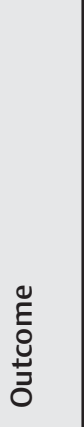 & 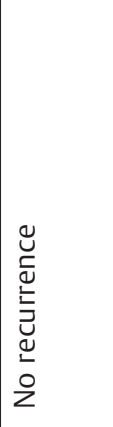 & \begin{tabular}{|l} 
㔛 \\
苋
\end{tabular} & 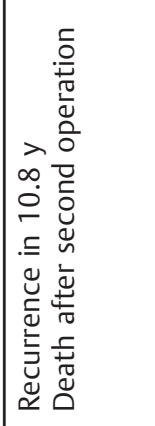 & 1 & 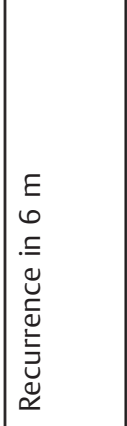 & 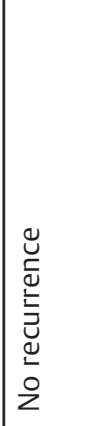 & 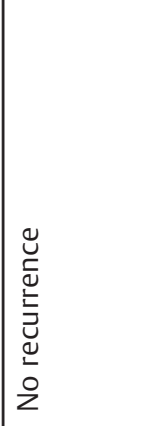 & 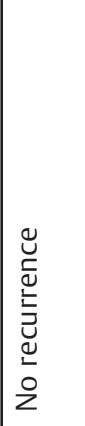 & 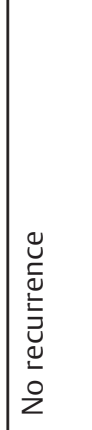 & 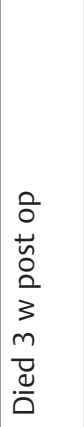 & 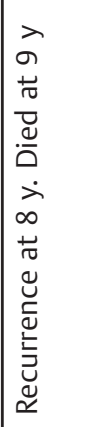 & 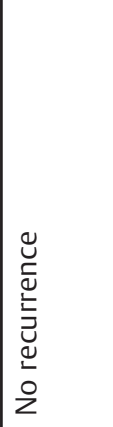 \\
\hline 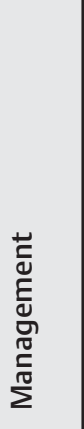 & 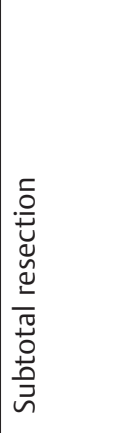 & 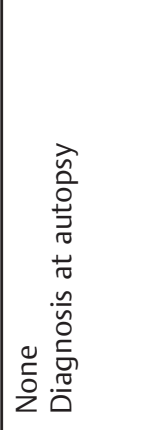 & 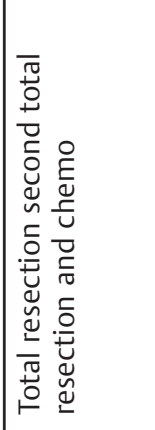 & 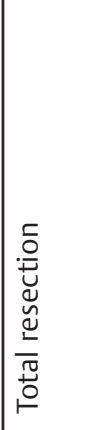 & 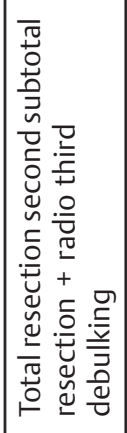 & 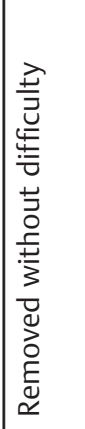 & 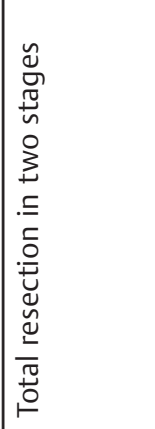 & 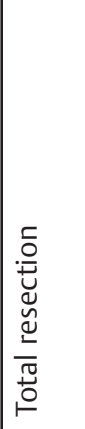 & 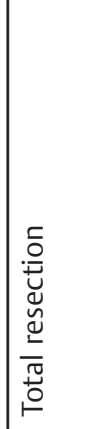 & 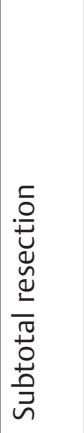 & 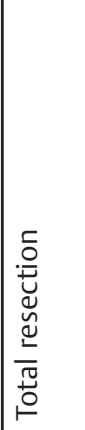 & 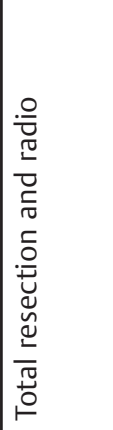 \\
\hline 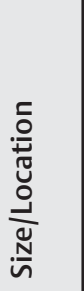 & 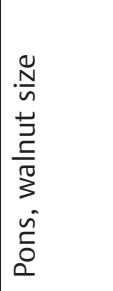 & 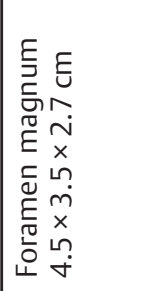 & 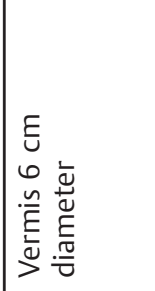 & 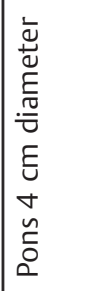 & 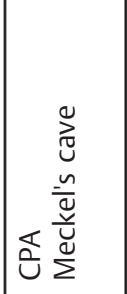 & 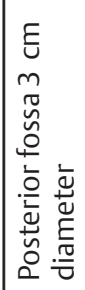 & 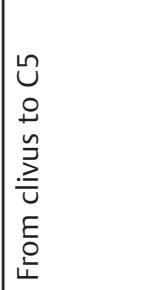 & 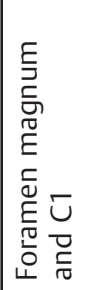 & ¿̇ & 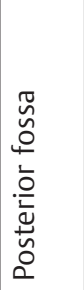 & 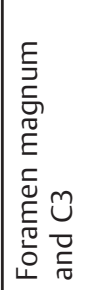 & 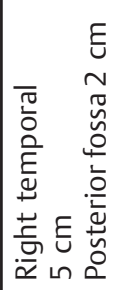 \\
\hline 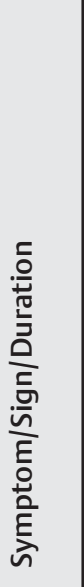 & 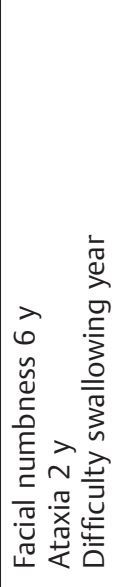 & 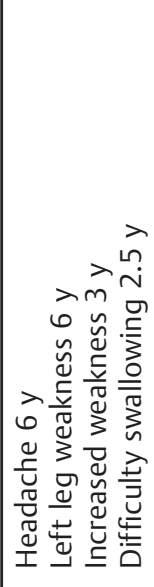 & 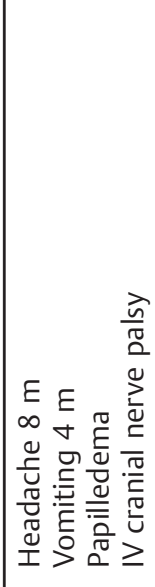 & 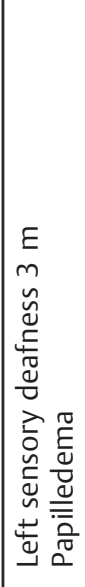 & 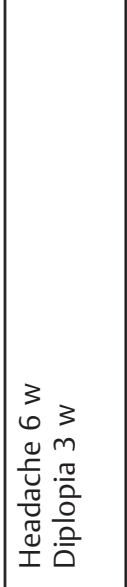 & 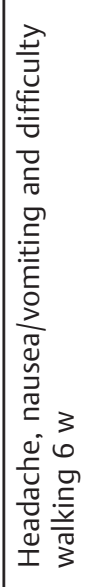 & 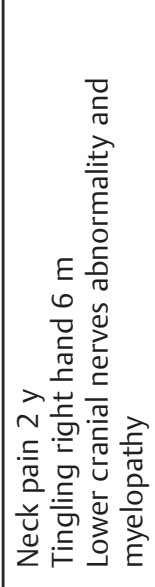 & 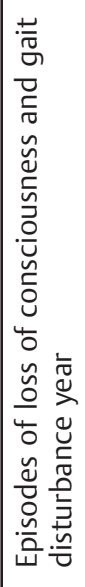 & 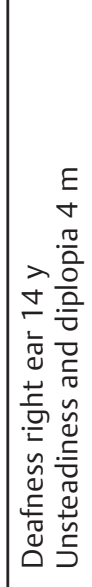 & 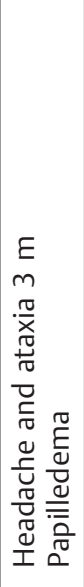 & 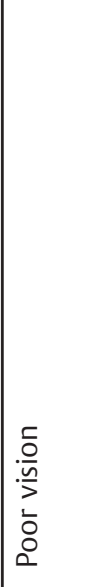 & 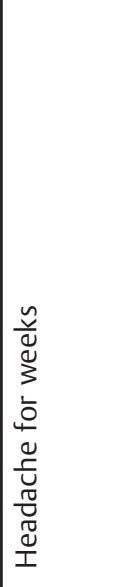 \\
\hline$\frac{\stackrel{x}{\tilde{u}}}{\frac{\tilde{g}}{\tilde{q}}}$ & $\begin{array}{l}\Sigma \\
\text { in }\end{array}$ & $\begin{array}{l}\Sigma \\
\pi\end{array}$ & $\begin{array}{l}L \\
N \\
N\end{array}$ & $\sum_{m}$ & $\sum_{G}$ & \begin{tabular}{|l}
$L$ \\
$\infty$ \\
$\infty$
\end{tabular} & $\begin{array}{l}\sum \\
\tilde{m}\end{array}$ & $\begin{array}{l}\Sigma \\
\tilde{W}\end{array}$ & $\begin{array}{l}4 \\
6\end{array}$ & $\frac{4}{\pi}$ & $\mid \begin{array}{l}\Sigma \\
g \\
q\end{array}$ & 岁 \\
\hline 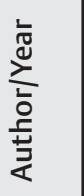 & 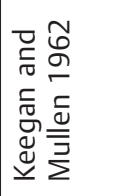 & 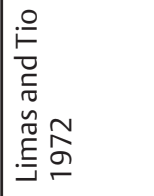 & 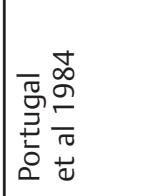 & 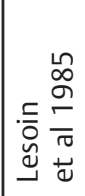 & 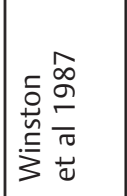 & 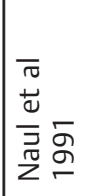 & 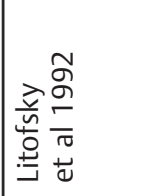 & 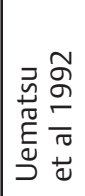 & 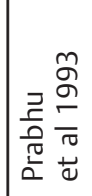 & 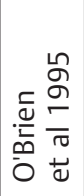 & & \\
\hline
\end{tabular}




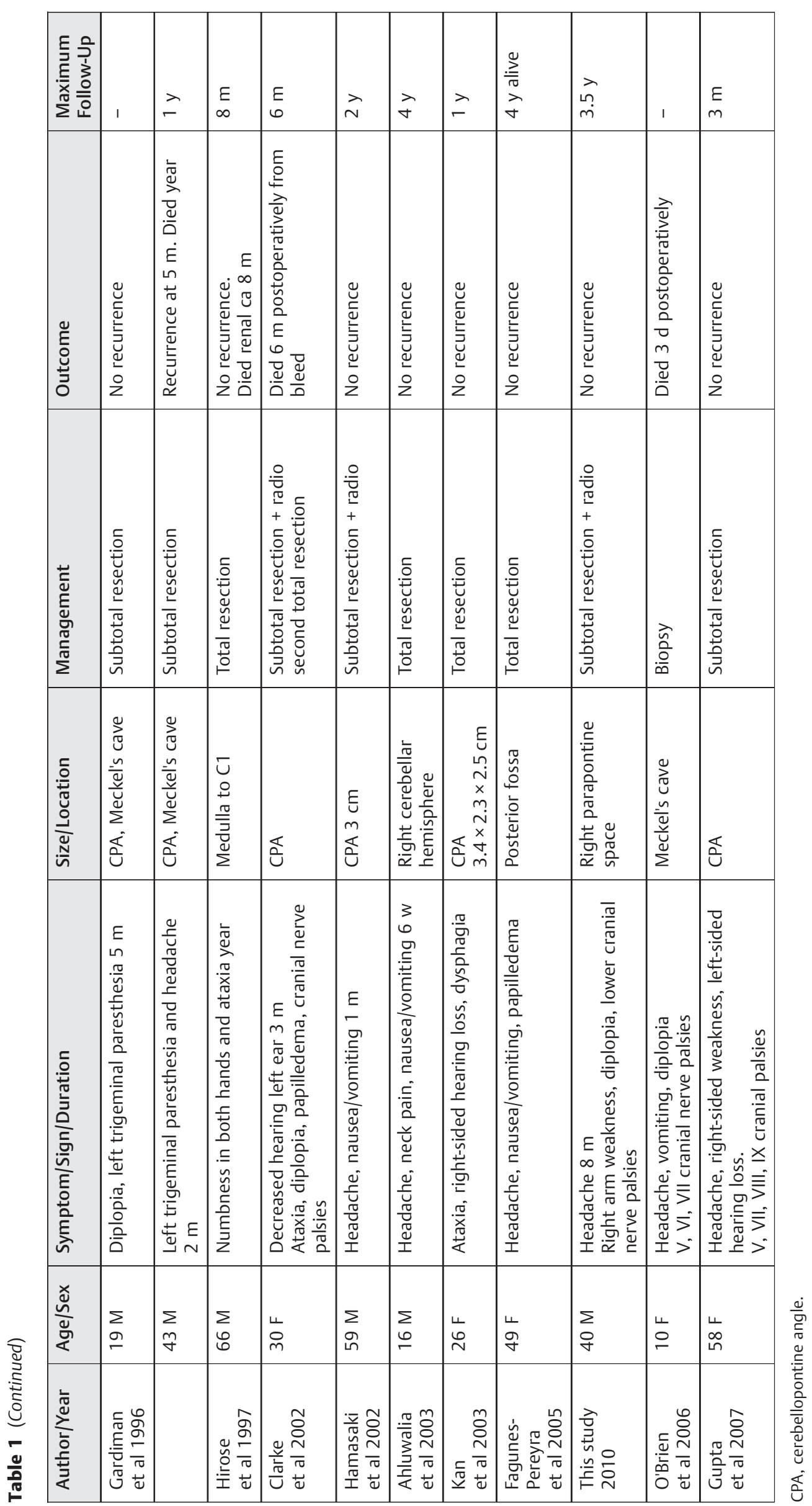


obstructive hydrocephalus or cerebellar dysfunction. ${ }^{6,7}$ These symptoms and signs are similar to other lesions in the same anatomical location, acoustic neuroma, meningioma, metastasis, and epidermoid cysts.

Although preoperative diagnosis was not made in all cases, a high index of suspicion is the key to clinch the diagnosis preoperatively. MRI characteristically demonstrated hyperintensity on T1-weighted images and hypo- or isointensity on T2-weighted images. ${ }^{6,8-10}$ These tumors enhanced homogenously with gadolinium. The lack of characteristic hyperintensity on T2-weighted imaging may possibly be due to their cellular or fibrous nature, resulting in diminished water content, paramagnetic effects of melanin, susceptibility artifacts or possibly hemorrhage.

The diagnosis is often made on immunohistochemistry and EM. PCPAMs are strongly immunoreactive to S-100 protein, HMB-45 and vimentin and, are nonreactive to epithelial membrane antigen and glial fibrillary acid proteins. $^{9,11-13}$ EM of melanocytomas demonstrate small smooth nuclei, indstinct nucleoli, abundant cytoplasm, and elaborate cytoplasmic processes. There is no external lamina around cytoplasmic membranes, no micropinocytotic vesicles and absent junctional complexes, and interdigitation of apposing cells. Our patient did not have EM examination because our pathologist was quite happy with the diagnosis without EM, however, if there was a doubt EM would be helpful. PCPAM must be distinguished from other histologically similar lesions of the central nervous system, such as melanotic schwannomas and meningiomas, ${ }^{9,11,14}$ metastatic or primary malignant melanoma, meningeal melanocytic naevi, pigmented neurofibromas, and pigmented primitive neuroectodermal tumors.

Although PCPAM and primary malignant melanomas of the leptomeninges originate from leptomeningeal melanocytes, they are different in appearance and behavior. ${ }^{14-19}$ PCPAMs have a much better prognosis than their malignant counterparts. Although most authors consider PCPAMs to be benign tumors, however melanocytomas in other locations such as the spinal cord tended to recur after resection and can be locally aggressive. ${ }^{9,12,20-24}$ As such some consider PCPAMs to be borderline tumors with guarded prognosis. ${ }^{9,12,21}$

Surgical resection had resulted in prolonged remission for up to 35 years, ${ }^{25}$ which justifies an aggressive surgical management. ${ }^{8,12,25,26}$ However, early recurrence was reported in $8 \%$ and late recurrence in $16 \%$ with fatal consequences. Incomplete resection invariably resulted into tumor progression with fatal consequences. Postoperative radiation therapy had been used in four patients; in one patient after complete resection who was alive and well 7 years after diagnosis, and in three patients after subtotal removal, one died from hemorrhage within 6 months, one recurred after 3 years and the third was alive and well 2 years after treatment. ${ }^{26,27}$ Hamasaki et al and Kurita et al treated patients with meningeal melanocytoma after partial surgical resection by radiosurgery with good early results. ${ }^{28,29}$ There was only one report in which chemotherapy was used in the management of spinal melanocytoma with time to tumor progression of 15 months. ${ }^{30}$ None of the reported cases metastasized implying that PCPAM are locally invasive tumors in which patients succumb to local recurrence and invasion rather than distant metastases.

\section{Conclusion}

PCPAMs are very rare tumors and often misdiagnosed preoperatively. They are generally benign tumors and welldifferentiated histologically. They should be considered in the differential diagnosis of tumors of the posterior cranial fossa. Complete surgical resection is associated with better outcome. The role of radiotherapy, radiosurgery, and chemotherapy is undetermined even when surgical resection was incomplete. Further documentation of these rare tumors and worldwide tumor registry is essential to study their behavior and find the best treatment paradigm.

\section{References}

1 Keegan HR, Mullan S. Pigmented meningiomas: an unusual variant. Report of a case with review of the litera ure. J Neurosurg 1962;19:696-698

2 Limas C, Tio FO. Meningeal melanocytoma ("melanotic meningioma"). Its melanocytic origin as revealed by electron microscopy. Cancer 1972;30(5):1286-1294

3 Portugal JR, Alencar A, Brito Lira LC, Carvalho P. Melanotic meningioma complicated by disseminated intravascular coagulation. Surg Neurol 1984;21(3):275-281

4 Lesoin F, Leys D, Pasquier F, et al. Melanotic meningiomas. Report of a case and review of the literature. Neurochir 1985;28(5): 205-207

5 Winston KR, Sotrel A, Schnitt SJ. Meningeal melanocytoma. Case report and review of the clinical and histological features. J Neurosurg 1987;66(1):50-57

6 Naul LG, Hise JH, Bauserman SC, Todd FDCT. CT and MR of meningeal melanocytoma. AJNR Am J Neuroradiol 1991;12(2): 315-316

7 Litofsky NS, Zee CS, Breeze RE, Chandrasoma PT. Meningeal melanocytoma: diagnostic criteria for a rare lesion. Neurosurgery 1992;31(5):945-948

8 Uematsu Y, Yukawa S, Yokote H, Itakura T, Hayashi S, Komai N. Meningeal melanocytoma: magnetic resonance imaging characteristics and pathological features. Case report. J Neurosurg 1992;76(4):705-709

9 Brat DJ, Giannini C, Scheithauer BW, Burger PC. Primary melanocytic neoplasms of the central nervous systems. Am J Surg Pathol 1999;23(7):745-754

10 Gupta A, Ahmad FU, Sharma MC, Garg A, Mehta VS. Cerebellopontine angle meningeal melanocytoma: a rare tumor in an uncommon location. Case report. J Neurosurg 2007;106(6): 1094-1097

11 O'Brien TF, Moran M, Miller JH, Hensley SD. Meningeal melanocytoma. An uncommon diagnostic pitfall in surgical neuropathology. Arch Pathol Lab Med 1995;119(6):542-546

12 Tatagiba M, Böker DK, Brandis A, Samii M, Ostertag H, Babu R. Meningeal melanocytoma of the $\mathrm{C} 8$ nerve root: case report. Neurosurgery 1992;31(5):958-961

13 Nestor SL, Perry A, Kurtkaya O, et al. Melanocytic colonization of a meningothelial meningioma: histopathological and ultrastructural findings with immunohistochemical and genetic correlation: case report. Neurosurgery 2003;53(1):211-214, discussion 214-215

14 Gardiman M, Altavilla G, Marchioro L, Boscolo L, Alessio L, Piazza M. Meningeal melanocytoma: a rare lesion of the central nervous system. Tumori 1996;82(5):494-496 
15 Hirose T, Horiguchi H, Kaneko F, et al. Melanocytoma of the foramen magnum. Pathol Int 1997;47(2-3):155-160

16 Clarke DB, Leblanc R, Bertrand G, Quartey GRC, Snipes GJ. Meningeal melanocytoma. Report of a case and a historical comparison. J Neurosurg 1998;88(1):116-121

17 Ahluwalia S, Ashkan K, Casey AT. Meningeal melanocytoma: clinical features and review of the literature. Br J Neurosurg 2003;17(4):347-351

18 Kan P, Shelton C, Townsend J, Jensen R. Primary malignant cerebellopontine angle melanoma presenting as meningioma: case report and review of the literature. Skull Base 2003; 13(3):159-166

19 Fagundes-Pereyra WJ, de Sousa L, Carvalho GT, Pittella JE, de Sousa AA. Meningeal melanocytoma of the posterior fossa: case report and literature review. Surg Neurol 2005;63(3):269-273, discussion 273-274

20 Koenigsmann M, Jautzke G, Unger M, Théallier-Janko A, Wiegel T, Stoltenburg-Didinger G. June 2002: 57-year-old male with leptomeningeal and liver tumors. Brain Pathol 2002;12(4):519-521

21 Córdoba A, Tuñón T, Vázquez JJ. [Meningeal melanocytoma. Presentation of a case and review of the literature]. Arch Neurobiol (Madr) 1989;52(2):93-99

22 Rades D, Heidenreich F, Tatagiba M, Brandis A, Karstens JH. Therapeutic options for meningeal melanocytoma. Case report. J Neurosurg 2001;95(2, Suppl):225-231
23 Bydon A, Gutierrez JA, Mahmood A. Meningeal melanocytoma: an aggressive course for a benign tumor. J Neurooncol 2003;64(3): 259-263

24 Ali Y, Rahme R, Moussa R, Abadjian G, Menassa-Moussa L, Samaha E. Multifocal meningeal melanocytoma: a new pathological entity or the result of leptomeningeal seeding? J Neurosurg 2009;111(3): 488-491

25 Prabhu SS, Lynch PG, Keogh AJ, Parekh HC. Intracranial meningeal melanocytoma: a report of two cases and a review of the literature. Surg Neurol 1993;40(6):516-521

26 Chow M, Clarke DB, Maloney WJ, Sangalang V. Meningeal melanocytoma of the planum sphenoidale. Case report and review of the literature. J Neurosurg 2001;94(5):841-845

27 Maiuri F, Iaconetta G, Benvenuti D, Lamaida E, De Caro ML. Intracranial meningeal melanocytoma: case report. Surg Neurol 1995;44(6):556-561

28 Hamasaki O, Nakahara T, Sakamoto S, Kutsuna M, Sakoda K. Intracranial meningeal melanocytoma. Neurol Med Chir (Tokyo) 2002;42(11):504-509(Tokyo)

29 Kurita H, Segawa H, Shin M, et al. Radiosurgery of meningeal melanocytoma. J Neurooncol 2000;46(1):57-61

30 Verma DS, Spitzer G, Legha S, McCredie KB. Chemoimmunotherapy for meningeal melanocytoma of the thoracic spinal cord. Report of a case. JAMA 1979;242(22):2435-2436 